\title{
Globe
}

Revue internationale d'études québécoises

\section{Anne Trépanier (dir.), La rénovation de l'héritage démocratique. Entre fondation et refondation, Ottawa, Les Presses de l’Université d'Ottawa, 2008}

\section{Marc Chevrier}

Volume 13, numéro 1, 2010

Culture et relations internationales

URI : https://id.erudit.org/iderudit/044652ar

DOI : https://doi.org/10.7202/044652ar

Aller au sommaire du numéro

Éditeur(s)

Globe, Revue internationale d'études québécoises

ISSN

1481-5869 (imprimé)

1923-8231 (numérique)

Découvrir la revue

Citer ce compte rendu

Chevrier, M. (2010). Compte rendu de [Anne Trépanier (dir.), La rénovation de l'héritage démocratique. Entre fondation et refondation, Ottawa, Les Presses de l'Université d'Ottawa, 2008]. Globe, 13(1), 192-196.

https://doi.org/10.7202/044652ar d'utilisation que vous pouvez consulter en ligne.

https://apropos.erudit.org/fr/usagers/politique-dutilisation/ 
récurrents entre Québec et Ottawa en matière de relations internationales et des conséquences de la mondialisation.

David Meren

Université Paris IV-Sorbonne/London School of Economics

\section{Anne Trépanier (dir.) \\ La rénovation de l'héritage démocratique. Entre fondation et refondation, Ottawa, Les Presses de l'Université d'Ottawa, 2008.}

Avec le temps, les livres collectifs se sont imposés comme la voie obligée de l'écriture et de la publication universitaires. Préparés à la suite d'un colloque dont ils mûrissent l'idée ou d'un appel de textes lancé pour en tester une, ces livres sont peut-être rarement lus d'une seule traite, et parfois ne sont pas faits pour être lus de la sorte. Les incongruités de construction d'un tel type de livre s'expliquent souvent par le procédé lui-même : un directeur de publication caresse un beau projet autour d'une idée prometteuse et convie une brochette d'auteurs dispersés à la gestation de textes dont il récolte la moisson, un peu comme un pêcheur jetant un filet à l'eau pour voir ce qu'elle contient. Or, d'ordinaire, ce sont les disparates entre les textes réunis qui affaiblissent un livre collectif; dans le cas de l'ouvrage dirigé par Anne Trépanier, qui prend soin d'indiquer qu'il n'est pas un collage, c'est le filet lui-même qui apparaît mal tissé, fait de mailles lâches et drôlement nouées, dans lequel sont pris des textes souvent intelligents et subtils, qui auraient mérité d'être autrement servis.

Le grand filet cousu par Anne Trépanier se déploie à peu près comme suit: la nation et la révolution, porteuses de transcendances radicales et tributaires de l'idée de fondation, sont à dépasser. Armons-nous du concept de refondation, «issue de la conceptualisation de l'idée de fondation, qui s'oppose à l'idée de révolution ", écrit Trépanier, pour l'appliquer à l'histoire canadienne-française devenue québécoise, à l'idée de liberté en général, puis, derechef, à l'histoire, ainsi qu'à l'identité politique, en particulier celles du Canada français hors Québec et des Autochtones. Beau programme en trois parties. Mais de quoi est-il question au juste? De refonder les bases politiques d'une communauté politique, par une action constituante, un moment de grande mobilisation collective, une revivifica- 
tion des assises morales et intellectuelles de la collectivité? Point du tout. La refondation n'est pas un acte politique, c'est une "entreprise mentale», nous assure Trépanier, de "remodelage", de gestion du rapport à l'héritage du passé, d'équilibrage des aspirations du groupe ou de "renouvellement» de son bagage imaginaire. Pour donner un peu de consistance au concept, l'auteur signe quatre textes dans un recueil en contenant douze, soit l'introduction et la conclusion, ainsi qu'un texte sur le moment prétendument refondateur qu'aurait été l'Acte de Québec de 1774 et un autre sur l'apparente réhabilitation des Patriotes dans le Québec contemporain. Au fond, ce que Anne Trépanier essaie de nous dire, c'est que les peuples qui ont raté leur révolution ou n'ont pu se donner de véritables moments fondateurs, comme le Québec, ne devraient pas s'en faire. Il est toujours possible aux acteurs historiques comme aux historiens eux-mêmes que désespèrent la défaite, la conquête, l'inaboutissement et le ratage collectifs, de se construire des vues de l'esprit édifiantes, conciliantes, réconciliantes, de s'octroyer des petites victoires et des raisons d'exister. En somme, la refondation, c'est de la réingénierie de la mémoire à des fins thérapeutiques, c'est une police d'assurance pour la paix sociale. Célébrant la réinvention de soi, $\mathrm{du}$ « rebrassage, $\mathrm{du}$ remix", la refondation a finalement un but politique, que l'auteure annonce dans son texte sur le choix du 21 mai comme fête nationale des Patriotes en 2001 : remodeler le trop-plein du passé du "groupe historique canadien du Québec» pour en extraire, par la distillation purifiante de l'herméneutique, les thèmes du vivre-ensemble post-national: l'universalité, la démocratie et la mixité du groupe - ou métissage. Toute à la recherche de "moments définiteurs d'identité», 1774 ou 2001, Anne Trépanier n'arrive pas vraiment à démontrer la valeur "heuristique» de son concept. Son étude du moment refondateur de 1774 est navrante de maladresse. Elle fait une exégèse banale et étriquée de deux pétitions adressées en 1764 et 1773 aux pouvoirs anglais, répétant les poncifs habituels sur le caractère féodal, traditionnel, absolutiste de la société canadienne. Manifestement, l'auteure semble n'avoir pas lu l'Acte de Québec lui-même pour lui faire dire que le Conquérant y reconnaît que le peuple canadien possédait son code civil propre - terme qui n'apparaît qu'en 1804 sous Napoléon. Il est également faux d'affirmer que cette reconnaissance contrevenait à la règle britannique de l'homogénéité des droits accordés aux Britanniques, car en fait, suivant le droit coutumier britannique, les peuples conquis par Sa Majesté conservent la jouissance de leurs coutumes et lois particulières. Dans sa conclusion, l'auteure ne prend point garde d'énoncer des erreurs d'interprétation en affirmant sans ambages que Stéphane Kelly et les auteurs de la nouvelle sensibilité historique ont 
embrassé l'idée de refondation dans leurs travaux. Accumulant les redites, les amalgames de concepts et les formules alambiquées ou pseudo-savantes, l'auteure essaie de greffer les réflexions des autres auteurs à la sienne, en vain.

Au vrai, les cinq textes qui suivent l'introduction d'Anne Trépanier pourraient former à eux seuls un livre distinct, bien éloigné du parti légitimiste et reconstructeur de mémoire dans lequel elle aurait aimé les enrôler. Quatre textes costauds posent à leur façon la question de la refondation, en histoire ou en politique, du Québec, sur des prémisses autrement plus solides. Éric Bédard examine les manières dont les historiens ont pu se représenter l'époque du Canada-Uni (1840-1867) comme un moment de fondation politique. Il constate que les études historiques actuelles sont dominées par un biais moderniste, qui subordonne l'analyse de l'histoire au repérage des brèches, des signes avant-coureurs de la modernité dans laquelle le Québec s'est engagé. À trop lire l'histoire à l'aune d'une telle téléologie, on risque de perdre de vue «la contingence dans laquelle les acteurs du passé ont pu baigner ». Au lieu de célébrer des avènements dont l'historien fait la genèse, Bédard préconise une autre écriture de l'histoire, axée sur la reconstitution de «moments» de quelques décennies où il s'agit de saisir «les concepts clés d'une délibération nationale». À juste titre, Bédard en appelle au renouvellement de l'histoire du politique au Québec, éclipsée par une trop grande insistance sur l'histoire sociale par l'école moderniste. Sébastien Socqué traite quant à lui de la querelle de l'ambiguïté chez les intellectuels québécois, à partir des célèbres réflexions d'Hubert Aquin sur la fatigue culturelle du Canada français. Socqué contraste la pensée d'Yvan Lamonde, qui se pose en archéologue flegmatique de cette ambivalence légendaire, avec celles de Gérard Bouchard, qui la fustige comme un attribut d'une pensée impuissante, et de Jocelyn Létourneau, qui la disculpe, par une refondation sémantique de l'histoire du Québec. Socqué souligne l'étrangeté de la thèse de Létourneau, radicale par son injonction et conservatrice par son souci de conserver cette ambivalence. À demi-mots, Socqué conclut que ces différentes tentatives de refondation historique ont suscité des résultats décevants et que la "question de l'ambiguïté, un peu artificielle», tire sa pertinence de la question de la nation. On relit avec grand plaisir l'article de Gilles Labelle, publié pour la première fois en 1998, sur le préambule de la déclaration de souveraineté de 1995, qui considère la fondation politique à une époque du désenchantement du monde où il n'est d'autorité légitime que fondée par elle-même. De tous les textes du recueil, c'est celui qui pose le plus clairement la question de la fondation - un peuple peut-il encore se fonder aujourd'hui par un acte inaugural? Après avoir relevé les silences et les 
présupposés de ce préambule, Labelle n'écarte toutefois pas toute possibilité de fondation pour l'avenir et entrevoit une fondation sur un mode républicain - en remettant à l'honneur la république bas-canadienne oubliée par le préambule de 1995 - qui inscrive la liberté et la division dans la chose commune à sauvegarder. Jean-Philippe Warren revient dans un autre texte sur son sujet favori : la pensée personnaliste au Québec. Warren met en lumière plusieurs des traits paradoxaux de cette pensée cherchant à fonder l'autorité sur la liberté de personnes authentiques, qui finira par vouloir déboulonner toute forme d'autorité institutionnelle qui entrave le mouvement de l'esprit et des choses. Le personnalisme rend compliquée toute fondation, car à force d'inviter la personne à se déprendre des routines et des formes sociales qui l'enferment, il ne peut concevoir de médiations qui guident la personne dans ses projets. Des adeptes du personnalisme comme Fernand Dumont finiront par être dépassés par le culte de la liberté qu'ils ont engendré et qui aboutit au vide d'une société pluraliste sans détermination. Warren voit dans le personnalisme de Dumont une philosophie «si large qu'elle finit par endosser tout et son contraire».

En deuxième partie de l'ouvrage, Stéphane Vibert et Philippe Corcuff explorent la question du rapport des Modernes à la fondation. Vibert reprend les pistes déjà tracées par Gilles Labelle en s'interrogeant sur les conditions de possibilités du "nous» dans les démocraties contemporaines, qui ont justement l'auto-fondation comme mythe fondateur. Si les démocraties semblent intrinsèquement hostiles à toute forme de fondation absolue ou définitive, elles ne supposent pas moins leur arrimage à un corps politique concret, voire un enracinement dans des institutions qui ont pour tâche de rendre possible la liberté démocratique. Les démocraties sont donc vouées à l'auto-limitation, à la reconnaissance de leur caractère "socialhistorique» et vivent alors précairement sur des fondations renouvelables, dans la conscience que leur projet d'autonomie politique ne peut être «ni fondé ni démontré logiquement et en raison ". Corcuff, au contraire de Vibert, semble se réjouir de cette précarité éclairée par des «Lumières tamisées" sous le règne des transcendances relatives, seules compatibles avec les sociétés individualistes. Se plaçant sous le magistère de Claude Lefort, John Dewey, Jacques Rancière, Sandra Laugier et Jacques Derrida, Corcuff multiplie les formules amusantes dont il parsème son texte proche de la plaisanterie philosophique. Il se présente ainsi en agnostique de la démocratie en se demandant si les citoyens ne trouvent pas plus désirables l'amour, la sexualité, le travail, le sport, l'art, la musique, le jeu, la lecture, la paresse, la gastronomie, etc. que la participation démocratique... 
En troisième partie, outre Anne Trépanier, Anne Gilbert et Michel Lavoie abordent respectivement la refondation de la communauté francoontarienne gagnée par le pluralisme identitaire dans ses écoles et le rôle du discours victimaire chez les Autochtones entre 1946 et 1998 dans la métamorphose de leur rapport à eux-mêmes et à l'État canadien. Ces deux textes ne manquent pas d'intérêt mais closent un livre inégal, sans véritable unité, parti d'une idée «refondatrice» somme toute peu féconde, qui nous apprend peu de choses sur le Québec comme sur notre époque.

Marc Chevrier Université du Québec à Montréal 